\title{
Contexto de elaboração do Plano de Imunização contra COVID-19 no Brasil
}

\author{
Backdrop to the development of Brazil's national COVID-19 \\ immunization plan
}

Laylla Ribeiro Macedo (https://orcid.org/0000-0002-6246-3559) ${ }^{1}$

Claudio Jose Struchiner (https://orcid.org/0000-0003-2114-847X) ${ }^{2}$

Ethel Leonor Noia Maciel (https://orcid.org/0000-0003-4826-3355) ${ }^{1}$

\footnotetext{
${ }^{1}$ Laboratório de Epidemiologia,

Universidade Federal do Espírito Santo. Av. Fernando Ferrari 514, Goiabeiras. 29075-910 Vitória ES Brasil. layllarm@hotmail.com ${ }^{2}$ Escola de Matemática Aplicada, Fundação Getúlio Vargas. Rio de Janeiro RJ Brasil.
}

\begin{abstract}
The process of developing Brazil's COVID-19 immunization plan began belatedly and involved a number of experts, including a technical group responsible for defining priority groups for vaccination. This process was permeated by contradictions between the government and researchers. Finally, on 20 January 2021, the government published an updated version of the plan, which remains limited in scope.

Key words Coronavirus infections, Immunization, Brazil
\end{abstract}

Resumo O processo de elaboração do Plano de Imunização contra Covid-19 no Brasil se iniciou tardiamente e contou com a participação de especialistas incluindo o grupo técnico responsável pela definição de grupos prioritários para a vacinação. Este processo foi permeado de indefinições entre o Governo Federal e pesquisadores e, finalmente, no dia 20 de janeiro de 2021 foi divulgada a versão atualizada do Plano ainda incipiente no que tange a sua abrangência.

Palavras-chave Infecções por coronavírus, Imunização, Brasil 
Após alguns meses do início da pandemia de COVID-19 e a partir da divulgação pelos laboratórios dos resultados dos ensaios clínicos dos imunobiológicos contra a doença, acirrou-se uma busca mundial pelos acordos comerciais a fim de adquirir a tão esperada vacina. Nesse contexto, o Governo do Brasil, mesmo que tardiamente, inicia o processo de construção de um Plano Nacional de Imunização contra a COVID-19 e convida especialistas para compor grupos de trabalho por áreas temáticas, incluindo pesquisadores responsáveis pela definição dos grupos prioritários para a vacinação baseados em evidências científicas.

Em paralelo a isso, tramitava no Supremo Tribunal Federal (STF) uma Arguição de Descumprimento de Preceito Fundamental (ADPF), ou seja, uma modalidade de ação que tem como finalidade o combate a atos desrespeitosos aos preceitos fundamentais da Constituição de $1988^{1}$, que no caso em questão resultou em uma exigência pela construção e divulgação pelo Governo de um Plano Nacional de Imunização.

Nesse momento, o planejamento do Ministério da Saúde (MS) considerava apenas o quantitativo de imunobiológicos que já demonstravam acordos de compra com o Governo, como o acordo com a Astrazeneca/Oxford, que prometia 100 milhões de doses e com a Covax Facility, que atenderia em torno de 20 milhões de doses, não contemplando integralmente os grupos prioritários previamente estabelecidos (aproximadamente 78 milhões de pessoas).

Sendo assim, no dia primeiro de dezembro de 2020, o Governo divulgou uma nota pública à imprensa, que relata sobre o processo de construção do Plano, suas fases e elege os grupos prioritários, incluindo a população privada de liberdade $(\mathrm{PPL})^{2}$. Essa nota gera uma reação do grupo apoiador do Governo, questionando a inclusão dessa população entre os grupos com prioridade.

Ressalta-se que esta questão reforça o desfavorecimento de alguns grupos populacionais específicos, demonstrando como a pandemia atinge de forma diferencial indivíduos que apresentam maiores vulnerabilidades de saúde, sociais e/ou ambientais, revelando questões vivenciadas historicamente e portanto evidenciadas ainda mais nesse momento de pandemia, apesar de exaustivamente registradas em estudos acerca dos determinantes sociais da saúde e suas consequências ${ }^{3}$.

Em reunião com o Governo no dia 9 de dezembro de 2020, foi apresentado aos especialistas que a população privada de liberdade seria excluída dos grupos prioritários, incluindo somente os trabalhadores do sistema prisional. Diante disso, os consultores integrantes do Grupo Técnico do "Eixo Epidemiológico do Plano Operacional de Vacinação contra COVID-19" publicaram uma Nota Técnica expressando que o Plano nesse formato apresentava um erro de concepção pois era inviável que o mesmo contemplasse apenas os dois acordos comerciais para compra de vacinas já estabelecidos com o Governo. A nota ainda destaca que um Plano Nacional deve alcançar todos os indivíduos que demandem da imunização, recomenda a inserção de todos os grupos prioritários pactuados e reforça os princípios do Sistema Único de Saúde (SUS) 4.

Em contradição à decisão do MS em excluir a PPL do Plano, estudos e relatórios indicam que essa população seja classificada como grupo de risco para evolução fatal da COVID-19, considerando principalmente as doenças frequentemente notificadas nesses indivíduos agravadas pelas condições preocupantes das unidades prisionais brasileiras. Assim, torna-se extremamente importante planejar estratégias para interromper a transmissão da COVID-19 com o objetivo de proteger a saúde das pessoas privadas de liberdade, dos trabalhadores penitenciários, dos profissionais de saúde e da sociedade em geral ${ }^{5}$.

Em resposta à ADPF mencionada, no dia 11 de dezembro de 2020, o ministro da Saúde apresentou ao STF a versão prévia do Plano Nacional de Operacionalização da Vacinação contra COVID- $19^{6}$. A fim de inferir legitimidade a esse Plano, no documento constavam os nomes dos especialistas consultores, porém sem a anuência dos mesmos, que então tomaram ciência desse novo documento e da exclusão de alguns grupos através da imprensa. Imediatamente os especialistas manifestaram uma reação pública, inclusive em redes sociais de grande alcance, o que resultou em uma ampla divulgação desse fato e fomentou um debate esclarecendo que, embora constasse os nomes dos especialistas no Plano, os mesmos não estavam de acordo com a retirada da população privada de liberdade e dos trabalhadores da educação, já que no Plano apresentado ao STF somente constavam os professores e não todos os trabalhadores da Educação. Sendo assim ratificado pelos especialistas novamente que o atual Plano não era condizente com a demanda real experimentada pela população brasileira.

Após esses acontecimentos, a primeira edição do Plano Nacional de Operacionalização da Vacinação contra COVID-19 foi divulgada pelo Governo no dia 16 de dezembro de 2020 apresentando a reinserção desses grupos ${ }^{7}$. Uma atualização do Plano ainda foi publicada no dia 20 de 
janeiro de 2021, Plano Nacional de Operacionalização da Vacinação contra COVID-19 - $2^{\circ}$ edição $0^{8}$, porém é importante esclarecer que, ainda assim, o Plano revisado somente contempla em torno de $40 \%$ da população do país, não obtendo o quantitativo idealmente necessário para al- cançar a imunidade de grupo. Sendo assim, até o presente momento o Brasil não apresentou a sua população um Plano Nacional de Imunização que de fato se mostre efetivo no controle da doença, mesmo diante do aumento do número de casos e óbitos registrados diariamente.

\section{Colaboradores}

LR Macedo trabalhou na concepção, redação do artigo e revisão crítica. ELN Macedo e CJ Struchiner trabalharam na concepção, revisão crítica e aprovação da versão a ser publicada.

\section{Agradecimentos}

Ao Conselho Nacional de Desenvolvimento Científico e Tecnológico (CNPq) e à Fundação Carlos Chagas Filho de Amparo à Pesquisa do Estado do Rio de Janeiro (Faperj). 


\section{Referências}

1. Brasil. Supremo Tribunal Federal (STF). 30 anos de Constituição. Arguição de Descumprimento de Preceito Fundamental - ADPF [Internet]. Brasília: STF; 2021 [acessado 2021 fev 9]. Disponível em: http://sistemas. stf.jus.br/30anosconstituicao/

2. Brasil. Agência Saúde. Assessoria de Comunicação do Ministério da Saúde. CORONAVÍRUS. Vacinação contra a Covid-19 será feita em quatro fases [Internet]. 2020. Brasília: Agência Saúde; 2021 [atualizado 2020 dez 4; acessado 2021 fev 9]. Disponível em: https:// www.gov.br/saude/pt-br/assuntos/noticias/vacinacao-contra-a-covid-19-sera-feita-em-quatro-fases

3. Figueiredo AM, Figueiredo DCMM, Gomes LB, Massuda A, Gil-García E, Vianna RPT, Daponte A. Determinantes sociais da saúde e infecção por COVID-19 no Brasil: uma análise da epidemia. Rev Bras Enferm 2020; 73(Supl. 2):e20200673.

4. Nota técnica. Considerações sobre vacinação contra a COVID-19 em populações vulneráveis e trabalhadores essenciais. Documento produzido pelo Grupo Técnico do Eixo Epidemiológico do Plano Operacional da Vacinação contra COVID-19. 2020.

5. Fundação Oswaldo Cruz (Fiocruz). COVID-19 e a população privada de liberdade [cartilha]. $1^{\text {a }}$ ed. Rio de Janeiro: Fiocruz; 2020.

6. Brasil. Ministério da Saúde (MS). Gabinete do Ministro. Coordenação-Geral do Gabinete do Ministro. Divisão de Análise Técnica de Documentos Oficiais. Ofício no 1499/2020/DATDOF/CGGM/GM/MS. Arguição de Descumprimento de Preceito Fundamental no 754; Brasília: MS; 2020.
7. Brasil. Ministério da Saúde (MS). Secretaria de Vigilância em Saúde. Departamento de Imunização e Doenças Transmissíveis. Coordenação Geral do Programa Nacional de Imunizações. Plano Nacional de Operacionalização da Vacinação contra a COVID-19. $1^{a}$ ed. Brasília: MS; 2020.

8. Brasil. Ministério da Saúde (MS). Secretaria de Vigilância em Saúde. Departamento de Imunização e Doenças Transmissíveis. Coordenação Geral do Programa Nacional de Imunizações. Plano Nacional de Operacionalização da Vacinação contra a COVID-19. $2^{\mathrm{a}}$ ed. Brasília: MS; 2021.

Artigo apresentado em 24/03/2021

Aprovado em 13/04/2021

Versão final apresentada em 15/04/2021

Editores-chefes: Romeu Gomes, Antônio Augusto Moura da Silva 